\title{
THE EFFECTS OF CONSTANT AND ALTERNATING TEMPERATURES ON THE REPRODUCTIVE POTENTIAL, LIFE SPAN, AND LIFE EXPECTANCY OF Anastrepha fraterculus (WIEDEMANN) (DIPTERA: TEPHRITIDAE)
}

\author{
CARDOSO, V. V., ${ }^{1}$ FERREIRA, M. P., ${ }^{1}$ MONTAGNER, J. M., ${ }^{1}$ FERNANDEZ, C. G., ${ }^{2}$ \\ MOREIRA, J. C. ${ }^{3}$ and OLIVEIRA, A. K. ${ }^{1}$ \\ ${ }^{1}$ Departamento de Genética, Instituto de Biociências, UFRGS, Av. Bento Gonçalves, 9500, C. P. 15053, \\ CEP 91501-970, Porto Alegre, RS, Brazil \\ ${ }^{2}$ Departamento de Ciências Morfológicas, Instituto de Ciências Básicas da Saúde, UFRGS, R. Sarmento Leite, \\ 500, CEP 90050-170, Porto Alegre, RS, Brazil \\ ${ }^{3}$ Departamento de Bioquímica, Instituto de Biociências, UFRGS, Av. Ramiro Barcelos, 2600, \\ CEP 91501-990, Porto Alegre, RS, Brazil \\ Correspondence to: Alice Kalisz de Oliveira, Departamento de Genética, UFRGS, Av. Bento Gonçalves, \\ 9500, C. P. 15053, CEP 91501-970, Porto Alegre, RS, Brazil, e-mail: alice.oliveira@ufrgs.br \\ Received August 28, 2001 - Accepted November 12, 2001 - Distributed November 30, 2002
}

(With 6 figures)

\begin{abstract}
Ovarian development, oviposition, larval eclosion, ornithine decarboxylase (ODC) activity, ovarian, testis and ejaculatory apodeme measurements (length, width, and area), and the number of spermatozoa of Anastrepha fraterculus (Wiedemann) were analyzed at alternating $\left(20^{\circ} / 6^{\circ} \mathrm{C}\right.$ and $\left.20^{\circ} / 13^{\circ} \mathrm{C}\right)$ and constant $\left(6^{\circ} \mathrm{C} ; 25^{\circ} \mathrm{C}\right)$ temperatures. Life span and life expectancy were also analyzed for both genders. All the results suggest that temperature, especially alternating temperatures, increase not only male and female reproductive potential but also their life span and life expectancy. These changes can be a powerful strategy triggered by $A$. fraterculus as a means to survive the stressful temperature conditions found in winter in the apple production region in Brazil, enabling this species to increase its population density and cause apple damage when spring begins.
\end{abstract}

Key words: reproductive potential, Tephritidae, temperature.

\section{RESUMO}

Efeitos de temperaturas constantes e alternadas no potencial reprodutivo, longevidade e expectativa de vida de fêmeas e machos de Anastrepha fraterculus (Wiedwmann) (Tephritidae)

O desenvolvimento ovariano, ovoposição, eclosão larval, atividade de ornitina descarboxilase (ODC), medidas de largura, comprimento e área do ovário, testículo e apódema ejaculatório e número de espermatozóides de Anastrepha fraterculus (Wiedemann) foram analisados em temperaturas alternadas $\left(20^{\circ} / 6^{\circ} \mathrm{C}\right.$ e $\left.20^{\circ} / 13^{\circ} \mathrm{C}\right)$ e constantes $\left(6^{\circ} \mathrm{C}\right.$ e $\left.25^{\circ} \mathrm{C}\right)$. A longevidade e a expectativa de vida também foram analisadas em ambos os sexos. Todos os resultados sugerem que a temperatura baixa e principalmente as temperaturas alternadas afetam não somente o potencial reprodutivo de machos e fêmeas, mas também sua longevidade e expectativa de vida, prolongando-as. Essas mudanças podem ser uma poderosa estratégia usada por A. fraterculus para sobreviver às condições estressantes de temperatura observadas no inverno na região produtora de maçãs, tornando-as capazes de aumentar sua densidade populacional e causar dano à maçã no início da primavera.

Palavras-chave: potencial reprodutivo, Tephritidae, temperatura. 


\section{INTRODUCTION}

Anastrepha (Schiner) is a native fly genus of the neotropical region with 195 known species, 94 of which are present in Brazil (Zucchi, 1999). Anastrepha fraterculus (Wied, 1830) is the most prevalent species, infesting more than 20 species of cultivated fruit in the states of Paraná, Santa Catarina and Rio Grande do Sul, amounting to 95\%97\% of the Tephritidae caught in traps (Salles \& Kovaleski, 1990). The cities of Vacaria (RS) and São Joaquim (SC) are characterized by large-scale apple production. A high rate of $A$. fraterculus oviposition in the earlier stages of apple development leads to the fall of the host fruit, causing severe apple production damage. Fletcher (1973) observed that only adult Dacus tryoni successfully overwinter. Thus, to maintain tolerable population frequency, control efforts are aimed at this developmental stage.

Insect development is largely determined by environmental temperature (Fletcher, 1989) and data collected by Humeres et al. (1996) showed the very low temperatures in the apple production region in the south of Brazil. Taufer (1998) and Taufer et al. (1998) analyzed fly ovarian development, life span, life expectancy, and some enzymatic patterns, especially those related to energy metabolism, at different ages of individuals reared at seasonal mean temperatures $\left(9^{\circ}, 13^{\circ}, 20^{\circ}\right)$ in this region, and at $25^{\circ} \mathrm{C}$ as control. They found that ovarian development only occurred at $25^{\circ} \mathrm{C}$ and $20^{\circ} \mathrm{C}$. Full ovarian development at $20^{\circ} \mathrm{C}$ needed twice the time than that required at $25^{\circ} \mathrm{C}$. The life span of the flies reared at $20^{\circ} \mathrm{C}$ was 31 weeks and 16 weeks at $25^{\circ} \mathrm{C}$. Flies kept at $9^{\circ} \mathrm{C}$ and $13^{\circ} \mathrm{C}$ did not show ovarian development but their life span increased. These data were obtained for flies kept at constant temperatures. However, it is known that the apple production region experiences temperature variations, especially in winter, the worst season for fly survival. Liu et al. (1995) showed that the development level in insects is higher at alternating than at constant temperatures. Thus, in our study, alternating and constant temperatures were used to analyze the reproductive potential, life span, and life expectancy of males and females. Furthermore, considering that ornithyne decarboxilase (ODC) is the key enzyme for polyamine synthesis and very important in tissue growth, cell division, and differentiation (Tabor \& Tabor, 1984), this enzyme was also analyzed in $30 \mathrm{~d}$ females. The resulting data can be used to develop more efficient methods of biological pest management since control with insecticides leads to an increase in the number of red acarids as a side effect.

\section{MATERIALS AND METHODS}

Infested fruit (Campomanesia xanthocarpa Berg.) were collected in the town of Vacaria, RS (28 $\left.30^{\prime} \mathrm{S} / 50^{\circ} 54^{\prime} \mathrm{W}\right)$, and kept in laboratory conditions at $25^{\circ} \mathrm{C} \pm 1^{\circ} \mathrm{C}, \mathrm{L}$ : D of $12: 12$ cycle, and 60-80RH. After adult eclosion, the F1 was distributed in plastic cages $(19 \times 13 \times 13 \mathrm{~cm})$ and provided with food (brown sugar: soy bean flour: wheat flour 3:1:1) and water. The cages were placed in two separate environmental climatic control chambers adjusted to $60-80 \mathrm{RH}$ and daily $12 / 12 \mathrm{~h}$. alternated temperatures $\left(20 / 6^{\circ} \mathrm{C} ; 20 / 13^{\circ} \mathrm{C}\right)$ corresponding to the maximal and minimal mean temperatures of winter and autumn respectively in the apple production area (Humeres et al., 1996). In addition, two other environmental climatic control chambers were adjusted to $60-80 \mathrm{RH}$ and constant temperatures of $6^{\circ} \mathrm{C}$ and $25^{\circ} \mathrm{C}$, conditions used as the control in all statistical comparisons. Five cages were used for each temperature.

\section{Ovarian development}

Five replicates of twenty fly couples in each cage were used for each temperature. One female from each cage at six different ages $(1,15,30,45,60$, and 90 days) was analyzed. The ovaries were dissected in physiological solution (Ephrussi); length, width, and area were measured under a Leica microscope $(5 \mathrm{x})$ coupled to a computer running Somniun, data processing software developed in IDL (Interactive Data Language) by the Laboratory of Vegetable Anatomy of the Federal University of Rio Grande do Sul. The ovarian measurements were analyzed by a non-parametric Kruskall-Wallis statistical test (Siegel, 1975). Afterwards, the ovarian inclusion was placed in glycol methacrylate resin and sectioned into $3 \mu \mathrm{m}$ slices in a Leica automatic microtome. This material was stained with hematoxylin-eosin and classified according to the ovarian development staging proposed by Bressan (1996): immature ovaries (IO); ovaries in development (DO) with first order oocytes in 
formation but no corionated oocytes; and mature ovaries (MO) with corionated oocytes.

\section{Oviposition and larval eclosion}

Five replicates were used for this analysis. Sixteen fly couples were placed in each plastic cage. Oviposition took place in synthetic fruit made with agar-agar $(8 \mathrm{~g})$, grape juice $(200 \mathrm{ml})$, and propionic acid $(1 \mathrm{ml})$. After cooling, these artificial fruits were wrapped with parafilm. Oviposition time was $24 \mathrm{~h}$. and the production of eggs and first instar larval eclosion was counted at eight different ages (30, $45,60,90,120,145,160$, and 180 days). The flies were fed as usual (brown sugar: soy bean flour: wheat flour 3:1:1) between age intervals. Fruit was not used for oviposition. Because females need an appropriate medium to oviposit, oviposition probably did not occur within cage walls.

\section{Ornithine decarboxylase activity}

Ornithine decarboxylase activity was measured in three samples of thirty ovaries of fifteen 30-day old females, replicated three times for the four temperatures. The $\mathrm{CO}_{2}$ released was determined by the method described by Wyatt et al. (1973) and protein content was measured using the method described by Lowry et al. (1951).

\section{Testis and ejaculatory apodeme measurements}

Five replicates of twenty fly couples from each cage were used for each temperature. Five males from each cage at six different ages $(1,15,30$, 45,60 , and 90 days of age) were analyzed. The testis and ejaculatory apodeme were placed in physiological solution (Ephrussi) and then measured (length, width, and area) and analyzed using the same procedure as that for females.

\section{Number of spermatozoa}

The reproductive potential was obtained by counting the number of moving spermatozoa present in the testis of five males from each of the five cages containing 20 couples and maintained at the various temperatures. The technical conditions developed by Camargo (1972) for Apis mellifera, later modified by Bressan (1995) for Anastrepha, were used. The testis were transferred to Becker and mashed in $1 \mathrm{~cm}^{3}$ of Bardahl (Rad Cool) 0.005\% solution. After shaking, one drop was placed in a NewBauer hemacytometer (1/400 SQ mm-1/1C deep) (Hirschmann EM Techcolor). The mean calculation for the number of spermatozoa was done by using the average of fifty units of hemacytometer ( $\mathrm{x}$ ) and the following equation:

$$
\begin{aligned}
& 0.004 \mathrm{~mm}^{3}-\mathrm{x} \\
& 1000 \mathrm{~mm}^{3}-\mathrm{y},
\end{aligned}
$$

$\mathrm{x}=\mathrm{X} / 0.004 \mathrm{~mm}^{3}$ and $\mathrm{y}=$ mean number of spermatozoa/1,000 $\mathrm{mm}^{3}$.

The data were analyzed by using the Tukey HSD statistical test.

\section{Life span and life expectancy}

Twenty-five A. fraterculus couples were placed in each of five cages for each temperature. The life span for females and males was observed weekly and life expectancy was calculated using the method proposed by Carey (1993). The "long rank" test (Soares \& Colosimo, 1995) was used to test the survival function equality.

\section{RESULTS}

\section{Female analysis}

The results for ovarian development showed that, at the age of 30 days and at $25^{\circ} \mathrm{C}, 60 \%$ of the ovaries were mature and $40 \%$ are in development while at other temperatures they were either DO or IO (Table 1). At the age of 90 days this situation is different because, while at $25^{\circ} \mathrm{C}, 100 \%$ of the ovaries are DO, at alternating temperatures they were $\mathrm{MO}$ or DO. At $6^{\circ} \mathrm{C}$, full ovarian development has never been observed.

The comparisons of length, width, and area of ovaries of females reared at $25^{\circ} \mathrm{C}$ and at the other temperatures were significantly different at $6^{\circ} \mathrm{C}$ except for length at 15 days, width at 90 days, and area at 45 days. Measurements in all experimental conditions were usually smaller than those of the controls, except at the age of 90 days where females reared at both alternating temperatures had larger ovarian areas than those of the controls.

The results for ovarian area are interesting when we compare all ages under the same temperature (Fig. 1). The area curve observed at $20 \% 6^{\circ} \mathrm{C}$ showed more or less the same shape as that observed at $25^{\circ} \mathrm{C}$, in spite of usually smaller values. It also seems that a larger difference between alternating temperatures was better for ovarian development since at $20^{\circ} / 13^{\circ} \mathrm{C}$ the ovaries showed small area values. 
TABLE 1

Ovarian developmental stage (MO = mature; DO = in development; IO = immature) and measurements (length, width, and area) of Anastrepha fraterculus, at different ages $(1,15,30,45,60$, and 90 days) in adult stage at alternating temperatures $\left(20^{\circ} \mathrm{C} / 6^{\circ} \mathrm{C}\right.$ and $\left.20^{\circ} \mathrm{C} / 13^{\circ} \mathrm{C}\right)$ and constant temperatures $\left(6^{\circ} \mathrm{C}\right.$ and $\left.25^{\circ} \mathrm{C}\right) . \mathrm{Data}^{\circ}$ obtained at constant $25^{\circ} \mathrm{C}$ was used as control in non-parametric Kruskall Wallis statistical analysis.

\begin{tabular}{|c|c|c|c|c|c|}
\hline $\begin{array}{c}\text { Ages } \\
\text { (in days) }\end{array}$ & $\begin{array}{c}\text { Temperature } \\
\left({ }^{\circ} \mathbf{C}\right)\end{array}$ & $\begin{array}{c}\text { Development } \\
(\%)\end{array}$ & $\begin{array}{l}\text { Length }(\mathrm{mm}) \\
(x \pm \mathrm{SE})\end{array}$ & $\begin{array}{l}\text { Width (mm) } \\
(x \pm \text { SE) }\end{array}$ & $\begin{array}{l}\text { Area } .10^{-5} \cdot\left(\mathrm{mm}^{2}\right) \\
\quad(x \pm \mathrm{SE})\end{array}$ \\
\hline 1 & 25 & $100 \mathrm{IO}$ & $0.0042 \pm 0.0003$ & $0.0028 \pm 0.0003$ & $0.0076 \pm 0.058$ \\
\hline \multirow{4}{*}{15} & 25 & $80 \mathrm{DO}: 20 \mathrm{IO}$ & $0.0047 \pm 0.0003$ & $0.0033 \pm 0.0002$ & $1.17 \pm 0.140$ \\
\hline & 6 & $100 \mathrm{IO}$ & $\begin{array}{c}0.0033 \pm 0.0033 \\
\text { ns }\end{array}$ & $\begin{array}{c}0.0024 \pm 0.0001 \\
p=0.009^{*}\end{array}$ & $\begin{array}{c}0.55 \pm 0.068 \\
\mathrm{p}=0.0009^{*}\end{array}$ \\
\hline & $20-6$ & 80 DO : 20 IO & $\begin{array}{c}0.00048 \pm 0.0003 \\
\text { ns }\end{array}$ & $\begin{array}{c}0.0031 \pm 0.0003 \\
\mathrm{~ns}\end{array}$ & $\begin{array}{c}1.13 \pm 0.190 \\
\mathrm{~ns}\end{array}$ \\
\hline & $20-13$ & $60 \mathrm{DO}: 40 \mathrm{IO}$ & $\begin{array}{c}0.0054 \pm 0.0002 \\
\mathrm{~ns}\end{array}$ & $\begin{array}{c}0.0036 \pm 0.0001 \\
\text { ns }\end{array}$ & $\begin{array}{c}1.40 \pm 0.070 \\
\mathrm{~ns}\end{array}$ \\
\hline \multirow{4}{*}{30} & 25 & $60 \mathrm{MO}: 40 \mathrm{DO}$ & $0.0080 \pm 0.0007$ & $0.0058 \pm 0.0008$ & $3.74 \pm 0.790$ \\
\hline & 6 & $80 \mathrm{IO}: 20 \mathrm{DO}$ & $\begin{array}{c}0.0052 \pm 0.0001 \\
p=0.016^{*}\end{array}$ & $\begin{array}{c}0.0033 \pm 0.0002 \\
p=0.016^{*}\end{array}$ & $\begin{array}{c}1.22 \pm 0.095 \\
\mathrm{p}=0.0090^{*}\end{array}$ \\
\hline & $20-6$ & $100 \mathrm{DO}$ & $\begin{array}{c}0.0053 \pm 0.0004 \\
p=0.047 *\end{array}$ & $\begin{array}{c}0.0035 \pm 0.0003 \\
\text { ns }\end{array}$ & $\begin{array}{c}1.48 \pm 0.270 \\
\mathrm{p}=0.047^{*}\end{array}$ \\
\hline & $20-13$ & $100 \mathrm{DO}$ & $\begin{array}{c}0.0051 \pm 0.0004 \\
p=0.028^{*}\end{array}$ & $\begin{array}{c}0.0034 \pm 0.0035 \\
\mathrm{p}=0.0090^{*}\end{array}$ & $\begin{array}{c}1.28 \pm 0.230 \\
\mathrm{p}=0.016^{*}\end{array}$ \\
\hline \multirow{4}{*}{45} & 25 & $60 \mathrm{MO}: 40 \mathrm{DO}$ & $0.01 \pm 0.0014$ & $0.0057 \pm 0.0005$ & $4.32 \pm 1.060$ \\
\hline & 6 & $80 \mathrm{IO}: 20 \mathrm{DO}$ & $\begin{array}{c}0.0062 \pm 0.0001 \\
p=0.0090^{*}\end{array}$ & $\begin{array}{c}0.0041 \pm 0.0002 \\
\mathrm{p}=0.0472 *\end{array}$ & $\begin{array}{c}1.90 \pm 0.090 \\
\mathrm{~ns}\end{array}$ \\
\hline & $20-6$ & $60 \mathrm{DO}: 40 \mathrm{MO}$ & $\begin{array}{c}0.0076 \pm 0.0060 \\
\text { ns }\end{array}$ & $\begin{array}{c}0.0049 \pm 0.0004 \\
\text { ns }\end{array}$ & $\begin{array}{c}3.54 \pm 0.690 \\
\text { ns }\end{array}$ \\
\hline & $20-13$ & $100 \mathrm{DO}$ & $\begin{array}{c}0.0057 \pm 0.0003 \\
p=0.009^{*}\end{array}$ & $\begin{array}{c}0.0037 \pm 0.0001 \\
\mathrm{p}=0.0090^{*}\end{array}$ & $\begin{array}{c}1.68 \pm 0.190 \\
\mathrm{p}=0.028^{*}\end{array}$ \\
\hline \multirow{4}{*}{60} & 25 & $80 \mathrm{MO}: 20 \mathrm{DO}$ & $0.0097 \pm 0.0008$ & $0.0058 \pm 0.0010$ & $4.53 \pm 0,54$ \\
\hline & 6 & $60 \mathrm{DO}: 40 \mathrm{IO}$ & $\begin{array}{c}0.0048 \pm 0.0004 \\
p=0.0090^{*}\end{array}$ & $\begin{array}{c}0.0031 \pm 0.0004 \\
\mathrm{p}=0.0090^{*}\end{array}$ & $\begin{array}{l}1.15 \pm 0.190 \\
\mathrm{p}=0.0009^{*}\end{array}$ \\
\hline & $20-6$ & $60 \mathrm{DO}: 40 \mathrm{MO}$ & $\begin{array}{c}0.0088 \pm 0.0002 \\
\text { ns }\end{array}$ & $\begin{array}{c}0.0048 \mathrm{~mm} \\
\mathrm{~ns}\end{array}$ & $\begin{array}{c}3.79 \pm 1.610 \\
n s\end{array}$ \\
\hline & $20-13$ & $100 \mathrm{DO}$ & $\begin{array}{c}0.0045 \pm 0.0001 \\
p=0.0090^{*}\end{array}$ & $\begin{array}{c}0.0030 \pm 0.0002 \\
p=0.0090^{*}\end{array}$ & $\begin{array}{l}1.04 \pm 0.096 \\
p=0.0090 *\end{array}$ \\
\hline \multirow{4}{*}{90} & 25 & $100 \mathrm{DO}$ & $0.0058 \pm 0.0003$ & $0.0033 \pm 0.0003$ & $1.42 \pm 0.200$ \\
\hline & 6 & $60 \mathrm{DO}: 40 \mathrm{IO}$ & $\begin{array}{c}0.0039 \pm 0.0002 \\
p=0.0090^{*}\end{array}$ & $\begin{array}{c}0.0024 \pm 0.0001 \\
\text { ns }\end{array}$ & $\begin{array}{c}0.63 \pm 0.052 \\
\mathrm{p}=0.0091^{*}\end{array}$ \\
\hline & $20-6$ & $60 \mathrm{MO}: 40 \mathrm{DO}$ & $\begin{array}{c}0.0083 \pm 0.0009 \\
\mathrm{~ns}\end{array}$ & $\begin{array}{c}0.0043 \pm 0.0004 \\
\text { ns }\end{array}$ & $\begin{array}{c}2.89 \pm 0.480 \\
\mathrm{p}=0.028^{*}\end{array}$ \\
\hline & $20-13$ & $80 \mathrm{DO}: 20 \mathrm{MO}$ & $\begin{array}{c}0.0068 \pm 0.0006 \\
\text { ns }\end{array}$ & $\begin{array}{c}0.0045 \pm 0.0006 \\
\text { ns }\end{array}$ & $\begin{array}{c}2.49 \pm 0.490 \\
p=0.0009^{*}\end{array}$ \\
\hline
\end{tabular}

ns $=$ not statistically different.

$* \mathrm{p}<0.05$. 


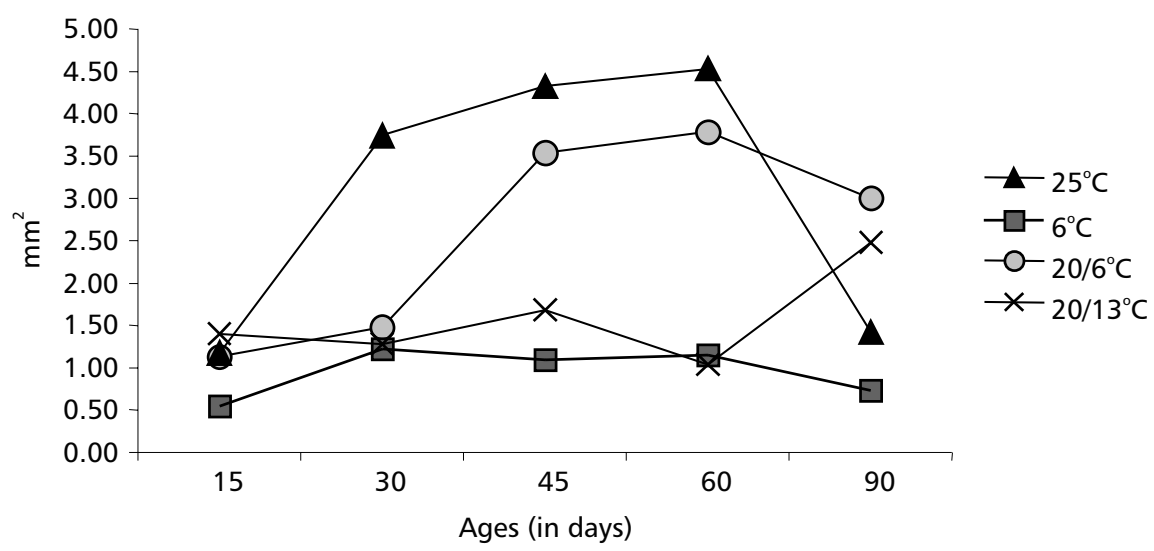

Fig. 1 - Ovarian area. $10^{-5}\left(\mathrm{~mm}^{2}\right)$ in different ages of Anastrepha fraterculus females reared at alternating $\left(20^{\circ} \mathrm{C} / 13^{\circ} \mathrm{C}\right.$; $\left.20^{\circ} \mathrm{C} / 6^{\circ} \mathrm{C}\right)$ and constant temperatures $\left(6^{\circ} \mathrm{C} ; 25^{\circ} \mathrm{C}\right)$.

When the same ages were compared at different temperatures we discovered that at 30 days in the experimental conditions, the ovarian area is significantly smaller than that of the control. This result is different at 90 days because at both alternating temperatures the ovarian area is larger than that of the control group.

Oviposition and larval eclosion are faster from day 30 to 45 at $25^{\circ} \mathrm{C}$, while at $20^{\circ} / 6^{\circ} \mathrm{C}$ these biological parameters showed higher numbers from day 90 to 180 . The performance at $20^{\circ} / 13^{\circ} \mathrm{C}$ is somewhat intermediate between those shown for the two previous conditions (Fig. 2).

When oviposition and larval eclosion at different temperatures but at the same age were compared, we found that at 30 days females reared at $25^{\circ} \mathrm{C}$ showed faster oviposition and eclosion, while females reared at alternating temperatures showed a lower rate of oviposition and eclosion. This behavior was seen until 45 days, but at 90 days the animals kept at alternating temperatures $(20 \%$ $6^{\circ} \mathrm{C}$ ) presented a higher rate of oviposition and larval eclosion. This pattern was constant until 180 days. Females reared at $20^{\circ} / 13^{\circ} \mathrm{C}$ presented an overall lower rate of oviposition and larval eclosion when compared to those shown for the other temperatures.

ODC activity (Fig. 3) was greater (115\%) in animals reared at $6^{\circ} \mathrm{C}$ than at $25^{\circ} \mathrm{C}(100 \%)$ and at alternating temperatures $\left(20 / 6^{\circ} \mathrm{C}=91 \% ; 20 / 13^{\circ} \mathrm{C}=\right.$ $106.5 \%)$. Therefore, increased ODC activity in female ovaries at $6^{\circ} \mathrm{C}$ can be related to the developmental stage, classified as immature (80\%) and in development (20\%) at 30 days when the ODC activity was measured.

The life span of females reared at alternating temperatures was longest, $336 \mathrm{~d}$ and $280 \mathrm{~d}$ at $20^{\circ} / 6^{\circ}$ and $20^{\circ} / 13^{\circ} \mathrm{C}$, respectively, when compared to the other two conditions, i.e., $182 \mathrm{~d}$ and $147 \mathrm{~d}$ at $25^{\circ}$ and $6^{\circ} \mathrm{C}$, respectively. The lowest value was found for females reared at $6^{\circ} \mathrm{C}$.

A higher life expectancy was also observed at $20^{\circ} / 6^{\circ} \mathrm{C}$ and $20^{\circ} / 13^{\circ} \mathrm{C}: 123.48 \mathrm{~d}$ and $13.65 \mathrm{~d}$ respectively. At $25^{\circ} \mathrm{C}$ and $6^{\circ} \mathrm{C}$ these values were $95.84 \mathrm{~d}$ and $68.12 \mathrm{~d}$, respectively.

The number of surviving female flies for each age group and temperature condition is shown in Fig. 4.

The long rank test for survival analysis showed significant results when the values for all temperature conditions were compared, except for the comparison between the alternating temperatures $\left(25^{\circ} \mathrm{C}\right.$ and $6^{\circ} \mathrm{C}: 26.46, \mathrm{df}=1, \mathrm{p}<0.001 ; 25^{\circ} \mathrm{C}$ and $20^{\circ} \%$ $6^{\circ} \mathrm{C}: 24.34, \mathrm{p}<0.001 ; 25^{\circ} \mathrm{C}$ and $20^{\circ} / 13^{\circ} \mathrm{C}: 12.49, \mathrm{p}<$ $0.001 ; 6^{\circ} \mathrm{C}$ and $20^{\circ} / 6^{\circ} \mathrm{C}: 68.68, \mathrm{p}<0.001 ; 6^{\circ} \mathrm{C}$ and $20^{\circ} / 13^{\circ} \mathrm{C}: 36.69, \mathrm{p}<0.001 ; 20^{\circ} / 6^{\circ} \mathrm{C}$ and $20^{\circ} / 13^{\circ} \mathrm{C}$ : $0.04, \mathrm{p}=0.850$ ).

\section{Male analysis}

The mean number of spermatozoa and measurements of length, width, and area of the $A$. fraterculus ejaculatory apodeme for males kept under the experimental and control conditions are shown in Table 2. 

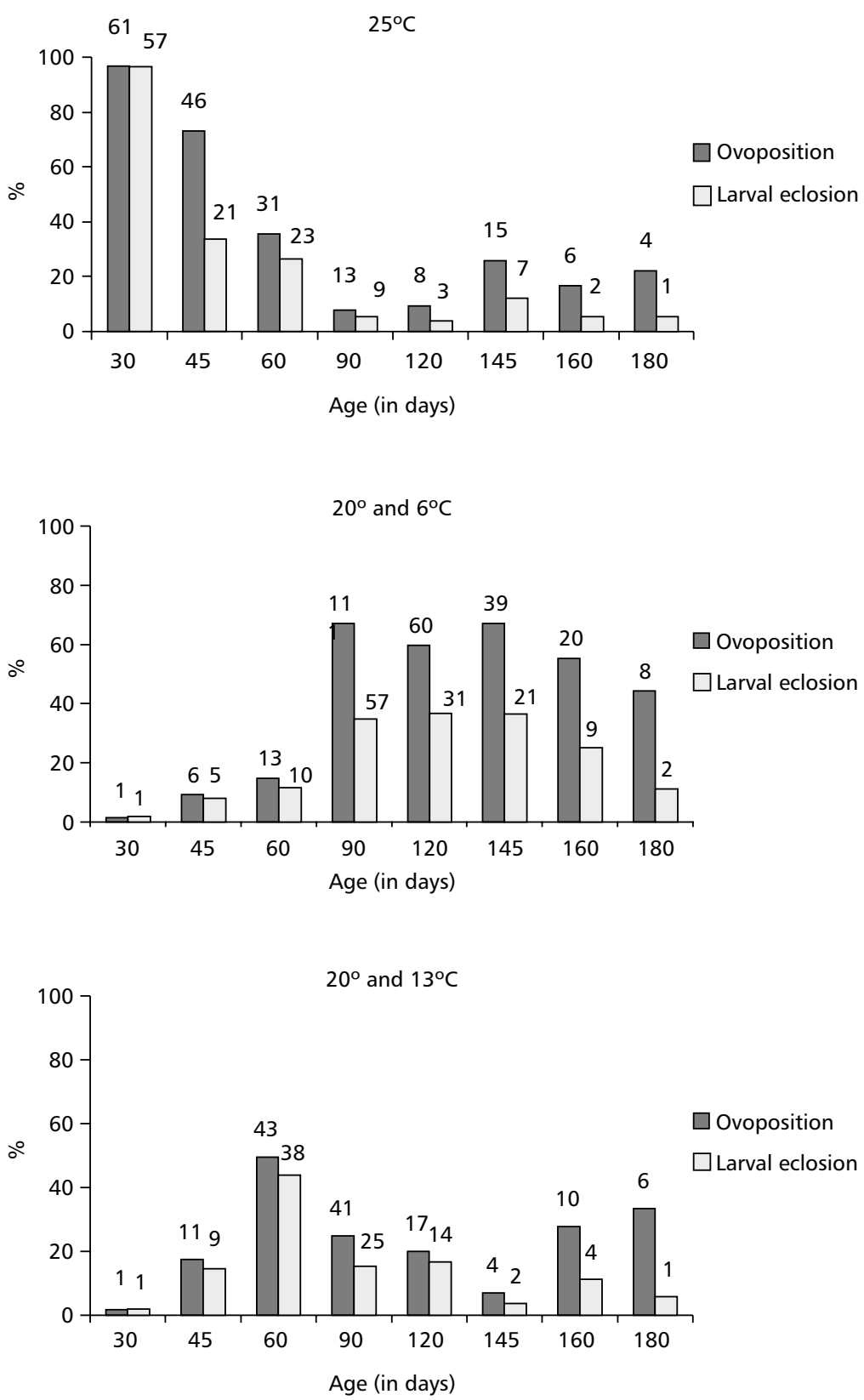

Fig. 2 - Oviposition and larval eclosion (\%) of Anastrepha fraterculus females reared at a constant temperature $\left(25^{\circ} \mathrm{C}\right)$ and alternating temperatures $\left(20^{\circ} \mathrm{C} / 13^{\circ} \mathrm{C} ; 20^{\circ} \mathrm{C} / 6^{\circ} \mathrm{C}\right)$ at different ages. Numbers above each bar correspond to the number of eggs laid. 


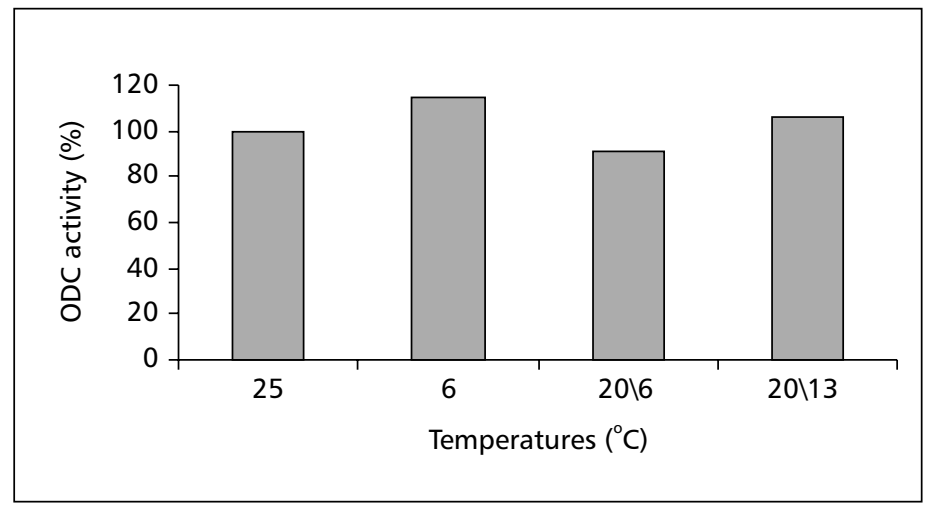

Fig. 3 - Ornithine decarboxylase activity(ODC) (\%) in 30-day-old females of Anastrepha fraterculus reared at alternating $\left(20^{\circ} \mathrm{C} / 13^{\circ} \mathrm{C} ; 20^{\circ} \mathrm{C} / 6^{\circ} \mathrm{C}\right)$ and constant temperatures $\left(6^{\circ} \mathrm{C} ; 25^{\circ} \mathrm{C}\right)$.

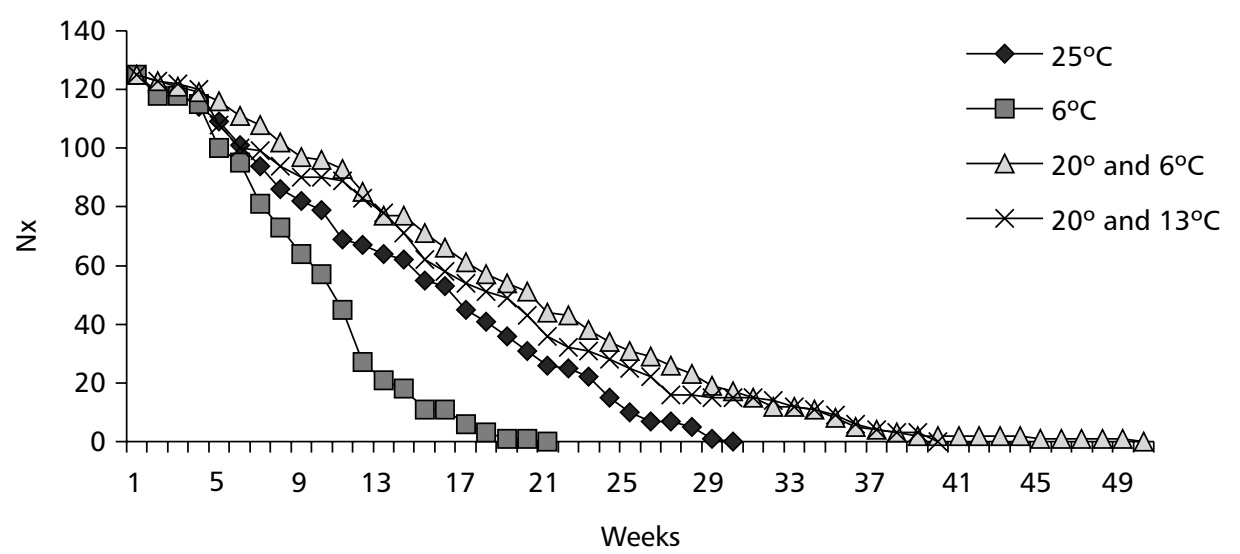

Fig. 4 - Number of surviving female Anastrepha fraterculus kept under different temperatures in relation to age.

As shown, 15-day-old males reared at $20 / 6^{\circ} \mathrm{C}$ showed a greater number of spermatozoa than control males kept at $25^{\circ} \mathrm{C}$. However, 30-day-old males in all temperature experimental conditions showed significantly fewer spermatozoa than the males in the control group. At the other ages (45, 60,90 days) there was no difference in the mean number of spermatozoa in all temperature conditions.

The Fig. 5 shows male performance for each temperature condition. A great number of spermatozoa was observed at 15 days under all conditions. However, while males at $25^{\circ} \mathrm{C}$ kept this number until day 30 , under other temperature conditions it decreased abruptly, especially at $20 \%$ $6^{\circ} \mathrm{C}$. Interesting is the slow decrease in the number of spermatozoa shown by males at $25^{\circ} \mathrm{C}$ and $6^{\circ} \mathrm{C}$, while males at alternating temperatures presented production peaks at 15 and 45 days. Thus, temperature fluctuation does not seem to be important for spermatozoa production after 45 days.

The measurements made of ejaculatory apodemes showed significant differences between males reared at $6^{\circ} \mathrm{C}$ and at $25^{\circ} \mathrm{C}$, except for length at 45 days (Table 2). At $6^{\circ} \mathrm{C}$, all measurements were less than those at $25^{\circ} \mathrm{C}$. However, the comparisons made between males at alternating temperatures and in the control group showed no difference. 
TABLE 2

Spermatozoa mean number (Y) and ejaculatory apodeme measures (length, width, and area) of Anastrepha fraterculus males, of different ages $(1,15,30,45,60$, and 90 days) reared in adult stage at alternated temperatures $\left(20^{\circ} \mathrm{C}\right.$ and $6^{\circ} \mathrm{C} ; 20^{\circ} \mathrm{C}$ and $\left.13^{\circ} \mathrm{C}\right)$ and constant temperature $\left(6^{\circ} \mathrm{C}\right.$ and $\left.25^{\circ} \mathrm{C}\right)$. Data obtained at constant $2^{\circ} \mathrm{C}$ were used as control in the Tukey HSD statistical test used for spermatozoa mean number and in non-parametric Kruskall Wallis statistical analysis of ejaculatory apodeme measures.

\begin{tabular}{|c|c|c|c|c|c|}
\hline $\begin{array}{c}\text { Ages } \\
\text { (in days) }\end{array}$ & $\begin{array}{c}\text { Temperature } \\
\left({ }^{\circ} \mathrm{C}\right)\end{array}$ & $\begin{array}{c}Y \\
(x \pm S E)\end{array}$ & $\begin{array}{l}\text { Legth }(\mathbf{m m}) \\
(x \pm \mathrm{SE})\end{array}$ & $\begin{array}{l}\text { Width }(\mathbf{m m}) \\
\quad(x \pm \mathrm{SE})\end{array}$ & $\begin{array}{c}\text { Area } .^{10^{-5}} \cdot\left(\mathrm{mm}^{2}\right) \\
(x \pm \mathrm{SE})\end{array}$ \\
\hline 1 & 25 & $37 \pm 1.61$ & $0.006 \pm 0.0003$ & $0.0021 \pm 0.00020$ & $1.06 \pm 0.060$ \\
\hline \multirow[t]{4}{*}{15} & 25 & $50 \pm 3.15$ & $0.0071 \pm 0.001$ & $0.0036 \pm 0.0001$ & $1.86 \pm 0.002$ \\
\hline & 6 & $45.6 \pm 2.45 \mathrm{~ns}$ & $\begin{array}{c}0.0058 \pm 0.001 \\
\mathrm{p}=0.009^{*}\end{array}$ & $\begin{array}{c}0.0022 \pm 0.001 \\
\mathrm{p}=0.009^{*}\end{array}$ & $\begin{array}{c}1.08 \pm 0.001 \\
\mathrm{p}=0.009 *\end{array}$ \\
\hline & $20-6$ & $\begin{array}{c}64 \pm 2.03 \\
p=0.01 *\end{array}$ & $0.0066 \pm 0.0023 \mathrm{~ns}$ & $\begin{array}{c}0.0029 \pm 0.0023 \\
\mathrm{p}=0.047^{*}\end{array}$ & $1.46 \pm 0.15 \mathrm{~ns}$ \\
\hline & $20-13$ & $54 \pm 3.59 \mathrm{~ns}$ & $0.0072 \pm 0.0002 \mathrm{~ns}$ & $0.0031 \pm 0.0035 \mathrm{~ns}$ & $1.78 \pm 0.055 \mathrm{~ns}$ \\
\hline \multirow[t]{4}{*}{30} & 25 & $50 \pm 2.79$ & $0.0079 \pm 0.00031$ & $0.0043 \pm 0.0001$ & $2.46 \pm 0.15$ \\
\hline & 6 & $\begin{array}{c}31 \pm 2.50 \\
\mathrm{p}=0.0001^{*}\end{array}$ & $\begin{array}{c}0.0062 \pm 0.0003 \\
p=0.009^{*}\end{array}$ & $\begin{array}{c}0.0028 \pm 0.0001 \\
\mathrm{p}=0.009^{*}\end{array}$ & $\begin{array}{c}1.25 \pm 0.905 \\
\mathrm{p}=0.009 *\end{array}$ \\
\hline & $20-6$ & $\begin{array}{c}27 \pm 2.10 \\
\mathrm{p}=0.0001 *\end{array}$ & $0.0079 \pm 0.0002 \mathrm{~ns}$ & $\begin{array}{c}0.0037 \pm 0.00019 \\
\text { ns }\end{array}$ & $2.13 \pm 0.67 \mathrm{~ns}$ \\
\hline & $20-13$ & $\begin{array}{l}36 \pm 2.52 \\
p=0.004 *\end{array}$ & $0.0072 \pm 0.0001 \mathrm{~ns}$ & $0.0040 \pm 0.0001 \mathrm{~ns}$ & $2.18 \pm 0.15 \mathrm{~ns}$ \\
\hline \multirow[t]{4}{*}{45} & 25 & $34 \pm 3.35$ & $0.0072 \pm 0.0002$ & $0.0043 \pm 0.0001$ & $2.23 \pm 0.095$ \\
\hline & 6 & $35 \pm 3.50 \mathrm{~ns}$ & $0.0062 \pm 0.0023 \mathrm{~ns}$ & $\begin{array}{c}0.003 \pm 0.0030 \\
p=0.0009 *\end{array}$ & $\begin{array}{c}1.46 \pm 0.0058 \\
\mathrm{p}=0.009 *\end{array}$ \\
\hline & $20-6$ & $48 \pm 4.41 \mathrm{~ns}$ & $0.0072 \pm 0.0003 \mathrm{~ns}$ & $0.0045 \pm 0.0003 \mathrm{~ns}$ & $2.12 \pm 0.169 \mathrm{~ns}$ \\
\hline & $20-13$ & $45 \pm 5.28 \mathrm{~ns}$ & $0.0073 \pm 0.0002 \mathrm{~ns}$ & $0.0040 \pm 0.0002 \mathrm{~ns}$ & $1.98 \pm 0.14 \mathrm{~ns}$ \\
\hline \multirow[t]{4}{*}{60} & 25 & $31 \pm 3.20$ & $0.0076 \pm 0.0003$ & $0.0043 \pm 0.0001$ & $2.39 \pm 0.076$ \\
\hline & 6 & $30 \pm 2.31 \mathrm{~ns}$ & $\begin{array}{c}0.0057 \pm 0.0025 \\
\mathrm{p}=0.00001^{*}\end{array}$ & $\begin{array}{c}0.0023 \pm 0.0001 \\
\mathrm{p}=0.009 *\end{array}$ & $\begin{array}{c}1.09 \pm 0.094 \\
\mathrm{p}=0.009 *\end{array}$ \\
\hline & $20-6$ & $31 \pm 2.65 \mathrm{~ns}$ & $0.0075 \pm 0.0003 \mathrm{~ns}$ & $0.0039 \pm 0.0039 \mathrm{~ns}$ & $2.22 \pm 0.171 \mathrm{~ns}$ \\
\hline & $20-13$ & $34 \pm 4.75 \mathrm{~ns}$ & $0.0070 \pm 0.001 \mathrm{~ns}$ & $0.0035 \pm 0.0003 \mathrm{~ns}$ & $\begin{array}{c}1.91 \pm 0.138 \\
\mathrm{p}=0.0163\end{array}$ \\
\hline \multirow[t]{4}{*}{90} & 25 & $22.5 \pm 4.50$ & $0.0074 \pm 0.003$ & $0.0039 \pm 0.0001$ & $1.93 \pm 0.085$ \\
\hline & 6 & $20 \pm 2.53 \mathrm{~ns}$ & $\begin{array}{c}0.0061 \pm 0.0004 \\
\mathrm{p}=0.00009 *\end{array}$ & $\begin{array}{c}0.0025 \pm 0.00009 \\
\mathrm{p}=0.009^{*}\end{array}$ & $\begin{array}{c}1.33 \pm 0.0012 \\
\mathrm{p}=0.009^{*}\end{array}$ \\
\hline & $20-6$ & $23 \pm 2.19 \mathrm{~ns}$ & $0.0074 \pm 0.0001 \mathrm{~ns}$ & $\begin{array}{c}0.00388 \pm 0.00016 \\
\text { ns }\end{array}$ & $2.06 \pm 0.00016 \mathrm{~ns}$ \\
\hline & $20-13$ & $17 \pm 2.33 \mathrm{~ns}$ & $\begin{array}{c}0.0074 \pm 0.0002 \\
\text { ns }\end{array}$ & $0.0036 \pm 0.0075 \mathrm{~ns}$ & $1.92 \pm 0.138 \mathrm{~ns}$ \\
\hline
\end{tabular}

$\mathrm{y}=$ mean number of spermatozoa $\mathrm{x} 106 / 1000 \mathrm{~mm}^{3} ; \mathrm{ns}=$ not statistically different; $* \mathrm{p}<0.05$. 


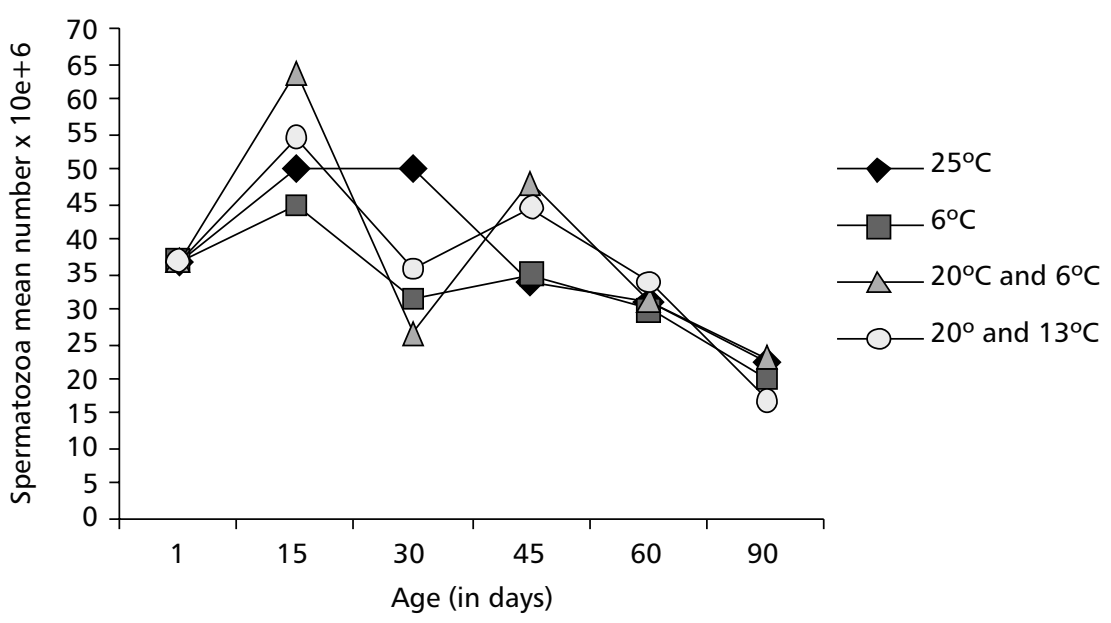

Fig. 5 - Spermatozoa mean number of Anastrepha fraterculus at different ages reared at alternating $\left(20 / 6^{\circ} \mathrm{C}\right.$ and $20 /$ $\left.13^{\circ} \mathrm{C}\right)$ and constant temperatures $\left(6^{\circ} \mathrm{C}\right.$ and $\left.25^{\circ} \mathrm{C}\right)$.

The results for testis measurements (length, width, and area) did not vary among different age groups and temperature conditions (mean length $=$ $0.0084 \pm 0.0001 \mathrm{~mm}$; mean width $=0.0035 \pm 0.0004$ $\mathrm{mm}$; and mean area $=2.28 \times 10^{-5} \pm 0.083 \mathrm{~mm}^{2}$ ). Therefore, the testes of $A$. fraterculus were nonprogressive.

Life span for males reared at $20 \% 6^{\circ} \mathrm{C}$ and $20 \%$ $13^{\circ} \mathrm{C}$ were $343 \mathrm{~d}$ and $273 \mathrm{~d}$ respectively, whereas at $25^{\circ} \mathrm{C}$ and $6^{\circ} \mathrm{C}$ they were $203 \mathrm{~d}$ and $140 \mathrm{~d}$ respectively. Furthermore, life expectancy at $20^{\circ} / 6^{\circ} \mathrm{C}$ and $20^{\circ} / 13^{\circ} \mathrm{C}$ was $119.31 \mathrm{~d}$ and $109.73 \mathrm{~d}$. Flies kept at $25^{\circ} \mathrm{C}$ and $6^{\circ} \mathrm{C}$ had life expectancies of $89.96 \mathrm{~d}$ and $57.54 \mathrm{~d}$ respectively.

The number of surviving flies for each age group is shown in Fig. 6.

The longest life span and highest life expectancy were shown by males reared at alternating temperatures, while males kept at low temperature $\left(6^{\circ} \mathrm{C}\right)$ showed the shortest/lowest life span/expectancy. Similar results were observed for females under the same temperature conditions.

The long rank test for survival analysis presented the same significant results as in the female group. Performance under all temperature conditions was different, except for the comparison between flies reared at alternating temperatures $\left(25^{\circ} \mathrm{C}\right.$ and $6^{\circ} \mathrm{C}: 41.61, \mathrm{p}<0.001 ; 25^{\circ} \mathrm{C}$ and $20^{\circ} / 6^{\circ} \mathrm{C}$ : $15.26, \mathrm{p}<0.001 ; 25^{\circ}$ and $20^{\circ} / 13^{\circ} \mathrm{C}: 7.68, \mathrm{p}<0.001$; $6^{\circ} \mathrm{C}$ and $20^{\circ} / 6^{\circ} \mathrm{C}$ : $83.82, \mathrm{p}<0.001 ; 6^{\circ} \mathrm{C}$ and $20^{\circ} / 13^{\circ} \mathrm{C}=$
$67.89, \mathrm{p}<0.001 ; 20^{\circ} / 6^{\circ} \mathrm{C}$ and $20^{\circ} / 13^{\circ} \mathrm{C}: 0.21, \mathrm{p}=$ $0.644)$.

The long rank test comparing the survival rate between the male and female groups at each temperature did not show significant results except for survival at $6^{\circ} \mathrm{C}\left(25^{\circ} \mathrm{C}: 0.53, \mathrm{p}=0.467 ; 6^{\circ} \mathrm{C}: 12.04\right.$, $\mathrm{p}<0.001 ; 20^{\circ} / 6^{\circ} \mathrm{C}: 0.06, \mathrm{p}=0.803 ; 20^{\circ} / 13^{\circ} \mathrm{C}: 0.82$, $\mathrm{p}=0.366)$.

\section{DISCUSSION}

Data collected from 1989 to 1994 by Humeres et al. (1996) on A fraterculus incidence in the apple production in southern Brazil showed no adults in winter when conventional traps were used. Since this fly begins its population increase in spring, three hypotheses, not mutually exclusive, can be posed: 1. migration of adults from regions where the temperature is not so cold; 2 . ontogenetic genetic regulation which allows flies to survive during winter; 3. inefficient traps.

The first hypothesis is difficult to accept since A. fraterculus has low dispersion ability (Kovaleski, 1997), but more appropriate methods could be used to test this hypothesis. The second and third hypotheses were corroborated by our results: $A$. fraterculus kept under alternating temperatures, an ordinary condition in the apple production region, showed delayed female and male reproductive potential and increased life span and life expectancy. 


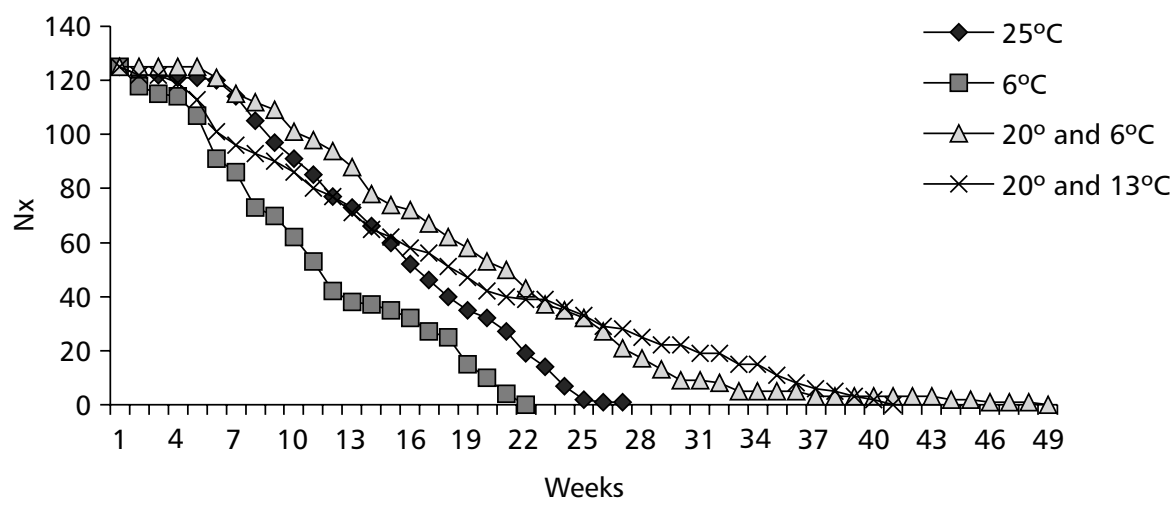

Fig. 6 - Number of surviving male Anastrepha fraterculus kept under different temperatures in relation to age.

The high incidence of mature ovaries until 90 days of age in alternating temperatures, especially at $20^{\circ} / 6^{\circ} \mathrm{C}$, is important for maintaining the fly population in the apple production region fields where temperature variations are common.

The flies kept at $6^{\circ} \mathrm{C}$ showed a shorter life span, lower life expectancy, lower ovarian and ejaculatory apodeme development, and greater ODC activity than the flies reared under the other temperature conditions. ODC is essential to polyamine synthesis and, as in Drosophila melanogaster (Meigen, 1830), ODC activity increases $24 \mathrm{~h}$ after female eclosion and decreases $48 \mathrm{~h}$ later, a pattern not seen in males. Birnbaum \& Gilbert (1990) suggested that polyamines act on ovarian maturation. Because ODC is an enzymatic step prior to polyamine synthesis, its increased activity can also indicate an absence of polyamines which results in the poor ovarian development found in the flies reared at $6^{\circ} \mathrm{C}$. Furthermore, Drew (1968) proposed that the ejaculatory apodeme needs to reach an optimal size to withstand the musculature used in copulation while Kamali \& Schultz (1974) hypothesized that sexual maturity is correlated with ejaculatory apodeme development. These three factors: low ovarian and ejaculatory apodeme development levels and high ODC activity can account for the absence of oviposition and larval eclosion in flies reared at $6^{\circ} \mathrm{C}$.

Another explanation for the results of ODC activity at $6^{\circ} \mathrm{C}$ could lie in a hypothesis presented by Wyatt et al. (1973). According to these authors, increase of this enzyme is due to a variety of stressful procedures, a phenomenon they called "injury syndrome". The low stress temperature $\left(6^{\circ} \mathrm{C}\right)$ would make females produce more ODC. In our experiment, the flies kept at $6^{\circ} \mathrm{C}$ also showed a shorter life span and lower life expectancy. These results suggest that the biological mechanisms used by flies to survive in the fruit area are limited at extreme temperatures and that only periods of alternating temperatures allow fly survival.

When males and females were compared, we found that the life span of males is longer at $25^{\circ} \mathrm{C}$ and $20^{\circ} / 6^{\circ} \mathrm{C}$ but shorter at $6^{\circ} \mathrm{C}$ and $20^{\circ} / 13^{\circ} \mathrm{C}$ while females showed a longer life span at the latter temperatures as well. However, female life expectancy was always higher than male life expectancy in all temperature conditions. The fact that females can retain spermatozoa is important for oviposition and larval eclosion and, consequently, for maintaining the fly population. Furthermore, since the longest life span and highest life expectancy were presented by both males and females at alternating temperatures, these circumstances can delay fly oviposition and eclosion and, therefore, explain the presence of some flies during springtime even after a severe winter. However, the number of flies in this case is small and this explains the results found by Humeres et al. (1996). More efficient traps would probably capture some flies. Meats (1983) analyzed the effects of alternating and constant temperature regimens in Dacus tryoni and observed that the damage of low temperatures is minimized when the organism is submitted to brief periods of less severe temperatures. 
The high rate of oviposition and larval eclosion observed at $90 \mathrm{~d}$ and $180 \mathrm{~d}$ in flies kept at alternating temperatures $\left(20^{\circ} / 6^{\circ} \mathrm{C}\right)$ corroborates the apparent diapause suggested by Baker \& Miller (1978) about the effect of cyclic and constant temperatures on the development of the fruit fly Rhagoletis cerasi.

The difference observed by us for life span and life expectancy in females reared at $20^{\circ} / 6^{\circ} \mathrm{C}$ and $25^{\circ} \mathrm{C}$ (for over two months) is related to their delayed ovarian development. It can explain the overwintering proposed by Kovaleski (1997) in the adaptation process of A. fraterculus colonization in the apple region of Vacaria (RS). Furthermore, it seems that the combination of two temperatures (alternating temperatures) decreased the effects of extreme temperatures by allowing animal acclimation.

Our findings on A. fraterculus behavior when reared under different temperature conditions and knowing of temperature conditions prior to apple growth (occurring in spring) can be used to predict fly oviposition and emergence, making it possible to foresee when damage will begin and, therefore, make better pest control choices, as suggested by Pritchard (1970).

Acknowledgments - We are grateful to Ricardo Pires for his help in computerized data analysis, to Dr. Adalécio Kovaleski (EMBRAPA-Vacaria, RS) for Anastrepha fraterculus supply, Fundação de Amparo à Pesquisa do Rio Grande do Sul (FAPERGS), and Conselho Nacional de Desenvolvimento Científico e Tecnológico (CNPq) for grants and fellowships.

\section{REFERENCES}

BAKER, C. R. B. \& MILLER, G. W., 1978, The effect of temperature on the post-diapause development on four geographical populations of the European cherry fruit fly (Rhagoletis cerasi). Entom. Exp. Appl., 23: 1-13.

BIRNBAUM, M. J. \& GILBERT, L. I., 1990, Juvenile hormone stimulation of ornithine decarboxilase activity during vitellogenesis in Drosophila melanogaster. J. Comp. Physiol. B., 160: 145-151.

BRESSAN, S., 1995, Desenvolvimento e potencial reprodutivo do macho de Anastrepha ssp. (Diptera: Tephritidae) em condições naturais. Rev. Bras. Ent., 39(4): 849-854.

BRESSAN, S., 1996, Desenvolvimento e potencial reprodutivo de fêmeas de Anastrepha obliqua (Diptera: Tephritidae) em condições naturais. Rev. Bras. Ent., 40: 11-16.
CAMARGO, C. A., 1972, Aspectos da reprodução dos Apídeos sociais. Dissertação de Mestrado, Faculdade de Medicina, Universidade de São Paulo, Ribeirão Preto, 266p.

CAREY, J. R., 1993, Applied demography for biologist, with special emphasis on insects. Oxford University Press, New York, 206p.

DREW, R. A. I., 1968, Morphology of reproductive system of Strumeta tryoni (Froggat) (Diptera: Thypetidae) with a method of distinguishing sexually mature adult males. J. Aust. Ent. Soc., 8: 21-32.

FLETCHER, B. S., 1973, The ecology of a natural population of the Queensland fruit fly, Dacus tryoni. Austr. J. Zool., 21: 541-565.

FLETCHER, B. S., 1989, Temperature-development rate relationships of the immature stages and adults of Tephritid fruit flies. In: A. S. Robson \& G. Hooper (eds.), Fruit flies: their biology natural enemies and control, 446p. Elsevier Science Publ., New York.

HUMERES, E., DA CRUZ, I. B., RIBEIRO, L. G. \& OLIVEIRA, A. K., 1996, Detection of Anastrepha fraterculus (Diptera: Tephritidae) adults in field under winter conditions in São Joaquim county, Brazil. Ann. 2nd Meeting of Working Group on Fruit Flies of Western Hemisphere, 1: 51.

KAMALI, K. \& SCHULTZ, T., 1974, Biology and Ecology of Gymnocarena diffusa (Diptera: Tephritidae) on Sunflkower in North Dakota. Ann. Entomol. Soc. Am., 67: 695-699.

KOVALESKI, A., 1997, Processos adaptativos na colonização de maçã (Malus domestica L.) por Anastrepha fraterculus (Wied) (Diptera: Tephritidae) na região de Vacaria, $R S$. Tese de Doutorado em Ciências, Universidade de São Paulo, SP, 122p.

LIU, S., ZHANG, G. \& ZHU, J., 1995, Influence of temperature variations on rate of development in insects: analysis of case studies from entomological literature. Ann. Ent. Soc. Am., 88: 107-119.

LOWRY, O. H., ROSENBROUGH, N. J., FARR, A. L. \& RANDALL, R. J. J., 1951, Protein measurement with the folin phenol reagent. Bio. Chem., 193: 265-275.

MEATS, A., 1983, Critical periods for developmental acclimation to cold in the Queesland fruit fly, Dacus tryoni. J. Insect Physiol., 29: 943-946.

PRITCHARD, G., 1970, The ecology of a natural population of the Queensland fruit fly, Dacus tryoni. III. The maturation of females flies in relation to temperature. Austr. J. Zool., 18: 77-89.

SALLES, L. A. B. \& KOVALESKI, A., 1990, Moscasdas-frutas em maciera e pessegueiro no Rio Grande do Sul. Hortisul, 1: 5-9.

SIEGEL, S., 1975, Estatística não paramétrica para ciências do comportamento. MacGraw-Hill, Rio de Janeiro, $350 \mathrm{p}$. 
SOARES, J. F. \& COLOSIMO, E. A., 1995, Métodos estatísticos na pesquisa clínica. 40 a Reunião Anual da Região Brasileira da Soc. Int. de Biometria. Minicursos. 105p. USP, Ribeirão Preto.

TABOR, C. W. \& TABOR, H., 1984, Polyamines. Ann. Rev. Biochem., 53: 749-790.

TAUFER, M., 1998, O efeito da temperatura na biologia de adultos de Anastrepha fraterculus (Diptera: Tephritidae): longevidade, desenvolvimento ovariano e isoenzimas relacionadas com o metabolismo energético. Dissertação de Mestrado, Curso de Pós-graduação em Genética e Biologia Molecular da UFRGS, 104p.

TAUFER, M., NASCIMENTO, J. C., CANTO, M. E. P., DA CRUZ, I. B. M. \& OLIVEIRA, A. K., 1998, Desenvolvimento ovariano, taxa de ovoposição e velocidade do desenvolvimento embrionário em Anastrepha fraterculus (Diptera: Tephritidae) quando submetidas a diferentes temperaturas. Anais do XVII Congresso Brasileiro de Entomologia, 1: 180.
WYATT, G. R., ROTHAUS, D., LAWLER, C. \& HERBST, E. J., 1973, Ornithine decarboxylase and polyamines in silkmouth pupal tissues: effects of ecdysones and injury. Biochem. Biophys. Acta, 304: 482-494.

ZUCCHI, R. A., 1999, Espécies de Anastrepha, Sinonímias, plantas hospedeiras e parasitóides. In: A. Malavasi \& R. A. Zucchi (eds.), Moscas-das-frutas de importância econômica no Brasil. Conhecimento Básico e Aplicado. 327p. Holos, Ribeirão Preto. 